Hydroécol. Appl. (2001) Tome 13 Vol. 1, pp. 37-55

\title{
Spéciation des contaminants métalliques en milieu estuarien : Intérêt de la modélisation et application au cadmium
}

\section{Speciation of metal contaminants in estuarine environment: Interest of modeling and application to cadmium}

\author{
J.L. Gonzalez, C. Dange et B. Thouvenin \\ IFREMER DEL Département "Polluants Chimiques" \\ Centre de La Seyne/mer Z.P. de Brégaillon BP 33083507 La Seyne/Mer cedex, FRANCE \\ E-mail : gonzalez@ifremer.fr
}

Résumé. - En milieu estuarien, le cadmium a un comportement de type non conservatif. II peut être présent sous différentes espèces : dissoutes (libre, complexes minéraux et organiques), colloïdales, particulaires (adsorbé, précipité...), ou intégrées par différents mécanismes dans les organismes. Cette distribution est le résultat de processus (physiques, chimiques et biologiques) qui sont contrôlés par de trés nombreux facteurs (salinité, $\mathrm{pH}, \mathrm{Eh}$, nature et concentration des matières en suspension, production primaire...). La modélisation géochimique est une approche très utile pour comprendre la dynamique de ce type de contaminant, notamment dans les milieux complexes que sont les estuaires. Un modèle de spéciation a été utilisé pour simuler les mesures effectuées ( $C d$ dissous et particulaire) lors de différentes campagnes réalisées dans les estuaires de la Seine, de la Loire et de la Gironde. Le modèle a été bâti à partir des connaissances acquises sur le terrain (mesures conjointes de $\mathrm{Cd}$ dissous et particulaire et des variables explicatives). Des expériences (en laboratoire et "in situ") ont éte nécessaires pour vérifier la validité des hypothèses du modèle et évaluer certains paramètres du modèle non mesurables directement (par exemple : cinétique de l'adsorption, densité de sites surface, constante de complexation des sites actifs, fraction particulaire non échangeable...).

La comparaison des résultats des simulations et des mesures indique que malgré ses simplifications, le modèle reproduit de façon satisfaisante le comportement de Cd lors du transit estuarien. Les écarts observés sont surtout liés à l'évaluation des paramètres de sorption des particules (notamment aux faibles salinités) et de la fraction de Cd particulaire échangeable (paramètre d'autant plus sensible que les teneurs en MES sont élevées). Dans un but prédictif, ce type de modèle doit être couplé à un modèle de transport hydrosédimentaire.

Mots-clés. - Cadmium, estuaire, spéciation, modélisation, complexation de surface. 


\begin{abstract}
In estuarine environment, cadmium has a nonconservative behavior. It can be present under various species: dissolved (mineral and organic complexes), colloidal and particulate forms (adsorbed, precipitated), or integrated by various mechanisms in the organisms. This distribution is the result of processes (physical, chemical and biological) which are controlled by many factors (salinity, $\mathrm{pH}, \mathrm{Eh}$, nature and concentration of suspended matter, primary production). Geochemical modeling is a very useful approach to understand the dynamics of this type of contaminant, especially in the complex systems which are the estuaries. A speciation model was used to simulate the measurements taken (dissolved and particulate $C d$ ) during various cruises carried out in the Seine, Loire and Gironde estuaries. The model was built starting from the knowledge obtained on the field. Experiments (in laboratory and "in situ") were necessary to check the validity of the assumptions of the model and to evaluate certain model parameters which cannot be measured directly.

The results of the validation indicate that in spite of its simplifications, the model reproduces in a satisfactory way the $\mathrm{Cd}$ dissolved / particulate distributions measured. With a predictive aim, this type of model must be coupled with a hydrosedimentary transport model.
\end{abstract}

Key words. - Cadmium, estuary, speciation, modeling, surface complexation.

\section{INTRODUCTION}

Du fait de leur position privilégiée à l'interface continent/océan et de leurs caractéristiques naturelles, les estuaires sont souvent très convoités par l'homme, aussi bien pour l'habitat, l'exploitation des ressources halieutiques que pour l'installation de ports et le développement d'activités agricoles et industrielles. A titre d'exemple, les estuaires de l'Escaut et de la Seine sont de "bonnes" illustrations de ces faits. Caractérisés par de très fortes densités de population, une industrialisation et une activité agricole parmi les plus importantes d'Europe, ces estuaires sont aussi les plus "contaminés". Toutes ces différentes activités anthropiques pourront avoir un impact notable, et à court terme, sur la structure et le fonctionnement de l'écosystème ce qui in- duira une diminution, voire une disparition, de la productivité naturelle et de l'intérêt socio-économique traditionnel de ces milieux.

Les milieux estuariens sont matériellement très accessibles mais les études que l'on peut y mener sont complexes et leurs résultats difficilement généralisables du fait de la forte variabilité spatio-temporelle des différents processus (physiques, chimiques et biologiques) qui les caractérisent. De plus, la mesure des niveaux de concentration des contaminants métalliques en estuaire est difficile. Se présentant à l'état de traces, leur étude nécessite l'utilisation de techniques d'échantillonnage "ultra-propres" et d'analyses complexes. La qualité de ces mesures est la seule garantie d'une exploitation des résultats obtenus, en termes d'évaluation de la contamination du 
milieu et de compréhension des mécanismes clés.

La plupart des métaux traces se rencontrent en milieu aquatique sous forme de différentes "espèces" (dissoutes: libre, complexes minéraux et organiques; associées à des colloïdes; particulaires: adsorbées, précipitées, intégrées à la matrice cristalline, associées au matériel biologique...). C'est la spéciation d'un contaminant qui va déterminer sa biodisponibilité et sa toxicité, mais les techniques actuelles, basées sur des critères opérationnels de séparation (filtration, ultrafiltration, extractions séquentielles...) ne permettent pas une "visualisation " directe des différentes espèces présentes dans le milieu.

Conjointement aux mesures réalisées sur le terrain, la modélisation mathématique du comportement des contaminants en milieu estuarien est un outil intéressant. Elle permet d'intégrer la grande variabilité spatio-temporelle du milieu et de prévoir les effets des modifications du système (augmentation des apports par le fleuve, rejets, changement des conditions physico-chimiques...) sur les flux du contaminant vers l'océan et sa spéciation. Mais ce type d'outil nécessite une bonne connaissance des processus majeurs (type de réaction d'échange dissous/particulaire, cinétique d'échange...) qui contrôlent la distribution de l'élément étudié et des variables clés qui les régulent (salinité, $\mathrm{pH}$, concentration en particules...). Dans notre étude, ces informations ont été recueillies lors de différentes campagnes réalisées dans les estuaires de la Seine, de la Loire et de la Gironde, ainsi qu'expérimentalement, grâce à l'utilisation (en laboratoire ou "in situ") de radioisotopes des contaminants métalliques étudiés $\left({ }^{109} \mathrm{Cd}\right.$ dans le cadre de cette étude). Cette approche a été entreprise, pour différents contaminants métalliques ( $\mathrm{Ag}, \mathrm{Co}, \mathrm{Cs}, \mathrm{Hg}$, $\mathrm{Pb}$ ), dans le cadre du projet "Modélisation du cycle des contaminants" de I'IFREMER et du programme Seine-Aval (Gonzalez et al., 1999 ; Thouvenin et al., 1999 ; Chiffoleau et al., 2001).

A titre d'exemple, nous présenterons la démarche réalisée pour le cadmium. Cet élément a un comportement non-conservatif assez bien connu en estuaire, ce qui nous a permis de choisir, à partir des nombreuses connaissances déjà acquises, les principaux processus à prendre en compte dans le modèle.

\section{SIMULATION DU COMPORTEMENT ET DE LA SPÉCIATION DU CADMIUM (MODĖLE MOCO)}

L'élaboration du modèle ("MOCO " : MOdèle de Complexation de surface) repose sur les connaissances acquises par le travail de terrain (processus dominants et variables clés) et des expériences en laboratoire (évaluation de paramètres non mesurables directement). La figure 1 résume la démarche qui a été suivie. 


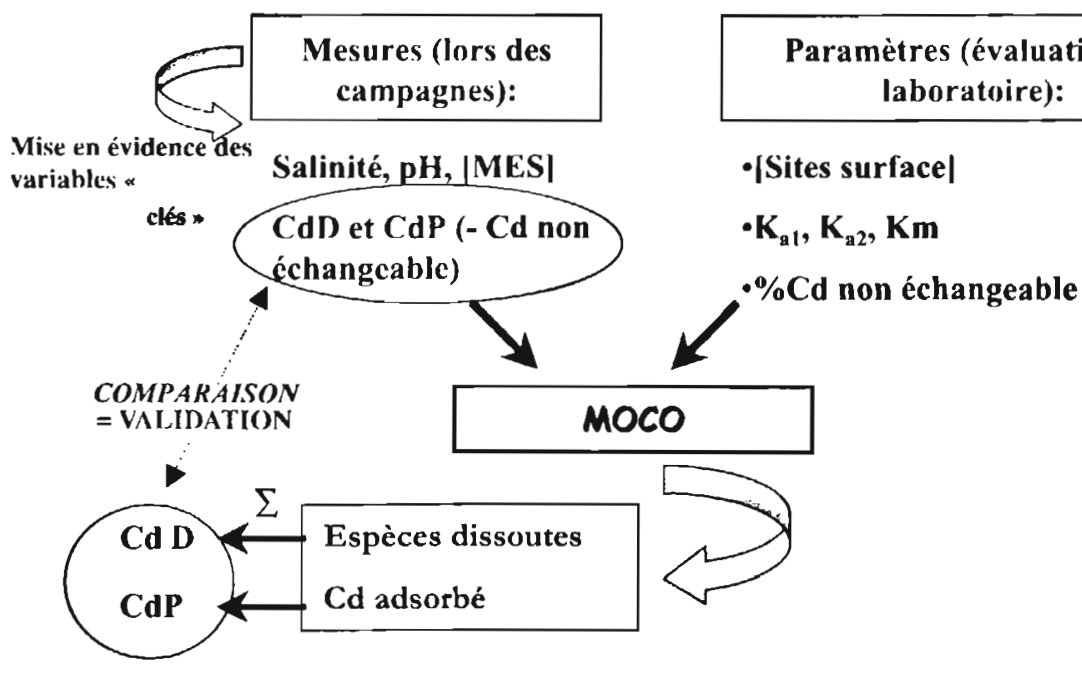

Fig. 1. - MOCO - Démarche, données nécessaires et validation.

Fig. 1. - MOCO - Processing, used data and validation.

\subsection{Principe et hypothèses du modèle}

En ce qui concerne le cadmium, l'une des caractéristiques communes à la plupart des estuaires macrotidaux est une augmentation puis une baisse des concentrations en Cd dissous en fonction de la salinité (Elbaz-Poulichet et al., 1987 ; Boutier et al., 1993 ; Chiffoleau et al., 1994). Au sein d'un même estuaire, ce maximum de $\mathrm{Cd}$ dissous (courbe "en cloche ") peut être observé pour différents régimes hydrauliques (crue, étiage) et à différentes saisons.

La plupart des études réalisées sur le terrain attribuent cette évolution caractéristique, à la désorption du cadmium lié aux particules entrant dans l'estuaire. La désorption est due à la formation de chlorocomplexes dissous très stables lorsque la chlorinité (salinité) augmente. Ce processus majeur a été confirmé par des expériences en laboratoire basées sur l'ajout de ${ }^{194} \mathrm{Cd}$ dans des échantillons résultant du mélange d'eau brute (phase dissoute + particules) de rivière et d'eau brute prélevée au large (Comans et Van Dijk, 1988 ; Turner, 1996). Le comportement général de cet élément à l'interface eau douceeau salée fait que les estuaires peuvent représenter des sources de $\mathrm{Cd}$ dissous non négligeables pour les eaux côtières adjacentes. Par exemple (fig. 2), le flux de Cd dissous sortant de la Seine vers l'océan est de l'ordre de 4 t/an (Chiffoleau et al., 1994) alors que celui qui entre dans l'estuaire est environ dix fois plus faible (Cossa et al., 1994). 


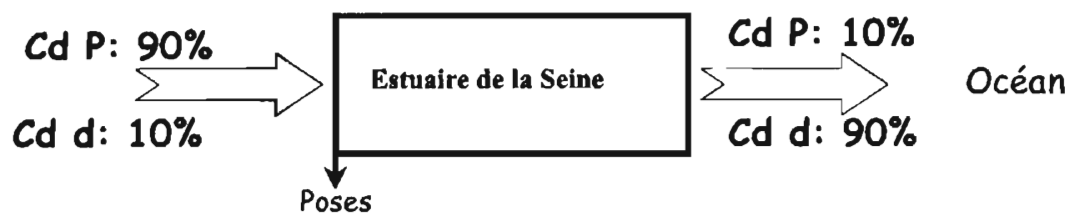

Fig. 2. - Importance des processus estuariens sur les flux de cadmium (en T/an) vers l'océan. Les estimations sont issues des travaux de Cossa et al., 1994 et Chiffoleau et al., 1994.

Fig. 2. - Importance of estuarine processes on cadmium fluxes (in T/year) towards the ocean. The estimates result from work of Cossa et al., 1994 and Chiffoleau et al., 1994.

La spéciation de Cd au cours du transit estuarien a été simulée grâce à un modèle de complexation de surface. Ce type de modèle traite l'adsorption de $\mathrm{Cd}$ sur les particules comme une formation de complexes avec des groupes fonctionnels de surface (de type hydroxyle). Les espèces dissoutes sont calculées à partir de la résolution des équilibres qui décrivent la complexation du métal avec différents ligands dissous (chlorures, hydroxydes, sulfates, composés organiques). Les principales réactions traitées par le modèle sont présentées dans la figure 3 . Le modèle permet, soit de prendre en compte différents constituants des particules naturelles (oxyhydroxydes, matière organique) en tant que phases adsorbantes individuelles, soit de traiter les particules globalement (capacités d'adsorption moyennes).

Le tableau I présente les réactions pouvant être prises en compte par le modèle. Dans ce travail, la formation de complexes organiques dissous n'est pas considérée et les particules sont traitées "globalement " (utilisation des propriétés moyennes de sorption évaluées sur des particules prélevées dans les différents estuaires). Le choix des processus repose sur les travaux portant sur la biogéochimie du $\mathrm{Cd}$ en milieu estuarien (Van der Weidjen et al., 1977 ; Comans et Van Dijk, 1988 ; Boutier et al., 1993 ; Chiffoleau, 1994 ; Turner, 1996 ; Krapiel et al., 1997 ; Boutier et al., 2000).

Les hypothèses et les simplifications faites dans cette approche sont les suivantes:

- les différentes réactions atteignent l'équilibre quasi-instantanément,

- les réactions d'adsorption et de désorption sont les processus majeurs (dans la colonne d'eau) responsables de la distribution dissous/particulaire de $\mathrm{Cd}$,

- l'adsorption est totalement réversible,

- la salinité, le pH et [MES] sont les variables qui dominent la spéciation de $\mathrm{Cd}$,

- les propriétés de surface des particules sont peu différentes tout le long de l'estuaire (leur intervalle de variation sera mesuré), 


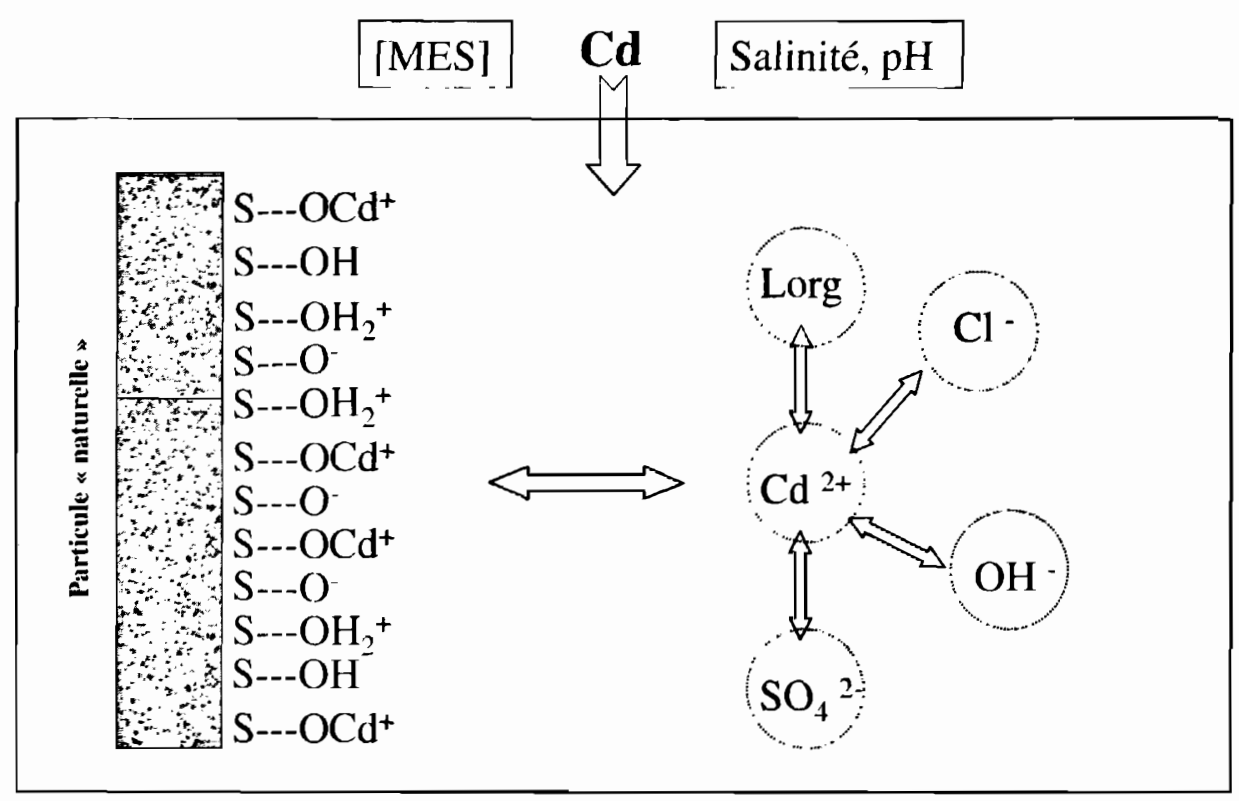

Fig. 3. - Processus traités par MOCO pour simuler la spéciation de $\mathrm{Cd}$ (Lorg= ligand organique dissous; $S=$ site de surface).

Fig. 3. - Processes taken into account by MOCO for the simulation of Cd speciation (Lorg= dissolved organic ligand; $S=$ surface site).

- la fraction colloïdale a peu d'influence sur la distribution de $\mathrm{Cd}$.

La validité de ces approximations a été évaluée par la comparaison des données de terrain aux résultats des simulations et grâce à des expérimentations. Les échantillons qui ont servi aux analyses de $\mathrm{Cd}$ dissous et particulaire (particules recueillies sur un filtre de $0,4 \mu \mathrm{m}$ ) ont été prélevés au cours de campagnes réalisées à différentes saisons et dans différentes conditions hydrologiques (crue, étiage). Les techniques d'échantillonnage, de filtration, de stockage et d'analyse spécifiques à l'étude des métaux traces sont décrites dans les travaux dont sont issues les mesures utilisées pour la validation du modèle (Boutier et al., 1993; Chiffoleau, 1994 ; Cossa et al., 1994 ; Boutier et al., 2000). Les campagnes de prélèvement sont des campagnes longitudinales au cours desquelles l'échantillonnage a été réalisé, en 2 ou 3 jours, le long du gradient de salinité des estuaires étudiés. Dans le cas des mesures effectuées à Poses, en limite amont de l'estuaire, l'échantillonnage mensuel a été effectué au point fixe pendant 28 mois (Cossa et al., 1994). 
Tableau I. - Processus pouvant être pris en compte par le modèle pour simuler la spéciation de Cd (dans cette étude, la complexation avec la MOD n'est pas considérée et les particules sont traitées de taçon globale).

Table I. - Processes which can be taken into account by the model to simulate the $\mathrm{Cd}$ speciation (in this study, the complexation with the DOM is not considered and the particles are treated in a global way).

Complexation du cadmium avec différents ligands dissous : Chaque équilibre est défini par une constante de complexation. Les constantes utilisées sont celles issues des travaux de Comans and Van Dijk, 1988 (valeurs prises pour une température de $25^{\circ} \mathrm{C}$ ). Les coefficients d'activité des différentes espèces ont été évalués en utilisant l'équation de Davies (Stumm, 1992).

$$
\begin{aligned}
& \mathrm{Cd}^{2+}+\mathrm{Cl}^{-}=\mathrm{CdCl}^{+} \\
& \mathrm{Cd}^{2+}+2 \mathrm{Cl}^{-}=\mathrm{CdCl}_{2} \\
& \mathrm{Cd}^{2+}+3 \mathrm{Cl}^{-}=\mathrm{CdCl}_{3}^{-} \\
& \mathrm{Cd}^{2+}+4 \mathrm{Cl}^{-}=\mathrm{CdCl}_{4}^{2-} \\
& \mathrm{Cd}^{2+}+\mathrm{OH}^{-}=\mathrm{Cd}(\mathrm{OH})^{+} \\
& \mathrm{Cd}^{2+}+2 \mathrm{OH}^{-}=\mathrm{Cd}(\mathrm{OH})_{2-} \\
& \mathrm{Cd}^{2+}+3 \mathrm{OH}^{-}=\mathrm{Cd}(\mathrm{OH})_{32-} \\
& \mathrm{Cd}^{2+}+4 \mathrm{OH}^{-}=\mathrm{Cd}(\mathrm{OH})_{4}{ }_{4-} \\
& \mathrm{Cd}^{2+}+\mathrm{SO}_{4}^{2-}=\mathrm{CdSO}{ }_{4} \\
& \mathrm{Cd}^{2+}+2 \mathrm{SO}_{4}^{2-}=\mathrm{Cd}\left(\mathrm{SO}_{4}\right)_{2}{ }^{2-} \\
& \mathrm{Cd}^{2+}+3 \mathrm{SO}_{4}^{2-}=\mathrm{Cd}\left(\mathrm{SO}_{4}\right)_{3-} \\
& \mathrm{Cd}^{2+}+4 \mathrm{SO}_{4}{ }^{2-}=\mathrm{Cd}\left(\mathrm{SO}_{4}\right)_{4}{ }_{4}
\end{aligned}
$$

$\left[\mathrm{Cl}^{-}\right](\mathrm{mol} / \mathrm{l})$ calculée à partir de la salinité

$[\mathrm{OH}](\mathrm{mol} / \mathrm{l})$ calculée à partir du $\mathrm{pH}$

$\left[\mathrm{SO}_{4}{ }^{2-}\right](\mathrm{mol} / \mathrm{l})$ mesurée ou calculée à partir de la salinité en supposant la conservativité

Complexation avec de la " matière organique dissoute" :

" $\mathrm{Cd}^{2+}+\mathrm{MOD}=\mathrm{CdMOD}$ *
[MOD] (mol/l) mesurée ou calculée à partir de la salinité en supposant la conservativité

Réactions de surface : Adsorption de Cd sur diffèrents types de phases adsorbantes ou à la surface d'une particule "globale". Les constantes de stabilité apparentes sont calculées à partir des constantes intrinseques en tenant compte des effets électrostatiques estimés par le modèle de Gouy-Chapman. (Stumm, 1992).

$$
\begin{aligned}
& \mathrm{S}-\mathrm{OH}_{2}{ }^{+}=\mathrm{S}-\mathrm{OH}+\mathrm{H}^{+} \mathrm{Ka} 1 \\
& \mathrm{~S}-\mathrm{OH}^{2}=\mathrm{S}-\mathrm{O}^{-}+\mathrm{H}^{+} \mathrm{Ka2}
\end{aligned}
$$

Sites "OXYDES"

$\mathrm{S}-\mathrm{OH}+\mathrm{Cd}^{2+}=\mathrm{S}-\mathrm{OCd}^{+}+\mathrm{H}^{+} \mathrm{Kox}$

Sites "ORGANIQUES"

$\mathrm{S}-\mathrm{OH}+\mathrm{Cd}^{2+}=\mathrm{S}-\mathrm{OCd}^{+}+\mathrm{H}^{+} \mathrm{Kor}$

Particule "globale"

$\mathrm{S}-\mathrm{OH}+\mathrm{Cd}^{2+}=\mathrm{S}-\mathrm{OCd}+\mathrm{H}^{+} \mathrm{Km}$
Ka1 et Ka2 sont les constantes d'acidité qui contrôlent la tendance des sites de surface à céder ou à capter des protons

Kox et Kor sont des constantes de complexation de surface déterminées expérimentalement (bibliographie)

La concentration en sites de surface (mol/) est calculée

à partir de la densité de sites $(\mathrm{mol} / \mathrm{g})$ de la phase considérée et sa teneur $(\mathrm{g} / \mathrm{g})$ mesurée dans les MES $(g / l)$

$\mathrm{Km}$ est déterminée expérimentalement. La concentration totale en sites de surface (mol/l) est déterminée d'après l'évaluation en laboratoire de la densitè de sites de surface $(\mathrm{mol} / \mathrm{g})$ et la concentration en MES $(\mathrm{g} / \mathrm{l})$ 


\subsection{Données nécessaires}

L'utilisation du modèle de complexation de surface (MOCO) nécessite la connaissance de différentes variables et d'un certain nombre de paramètres relatifs aux propriétés de sorption des particules naturelles (tableau I). Il est à noter que certains paramètres (les propriétés des sites de surface, la fraction de Cd particulaire "échangeable") n'étant pas mesurables directement, différentes techniques de laboratoire, décrites plus précisément dans Gonzalez et al. (1999; 2001), sont nécessaires pour leur évaluation.

\section{Ligands dissous :}

Les concentrations des différents ligands dissous (tableau I) sont issues des mesures réalisées conjointement aux analyses de Cd (chlorures d'après la salinité, hydroxydes d'après le $\mathrm{pH}$ ). Quand des mesures ne sont pas disponibles, du fait de leur quasi-conservativité dans les estuaires étudiés, la concentration en sulfates est calculée à partir de la salinité (connaissant les concentrations aux limites fluviales et marines de chaque estuaire).

\section{Ligands particulaires :}

Le calcul de la concentration en sites de surface susceptibles de complexer $\mathrm{Cd}$ est réalisé à partir de la teneur en MES mesurée et de la concentration en sites actifs des particules. Ce paramètre est évalué d'après la concentration des sites de surface échangeurs de protons (en mol g ' de MES) déterminée par titra- tion acido-basique des particules (Stumm, 1992 ; Gulmini et al., 1996).

\section{Propriétés des sites de surface} (tableau I) :

La valeur moyenne des constantes acido-basiques des sites de surface $\left(K_{i 1}\right.$ et $\left.K_{\mathrm{a} 2}\right)$ est déterminée par l'ajustement des résultats des titrations potentiométriques grâce au logiciel FITEQL (Westall, 1982 ; Gulmini et al., 1996 ; Wang et al., 1997).

La constante "globale" de complexation de ces sites $(\mathrm{Km})$ vis-à-vis de Cd est évaluée, sur des particules collectées dans les différents estuaires, grâce aux expériences basées sur l'utilisation de ${ }^{109} \mathrm{Cd}$ (Gonzalez et al., 1999 ; 2001).

\section{Fraction de Cd particulaire} "échangeable":

Cette évaluation est importante car :

- Le cadmium particulaire calculé par MOCO représente uniquement la fraction adsorbée à la surface des particules. Cette fraction ne peut être comparée directement au cadmium particulaire mesuré qui est constitué d'une fraction échangeable (potentiellement désorbable dans les conditions physico-chimiques rencontrées dans les estuaires) et d'une fraction non-échangeable (Cd incorporé dans la matrice cristalline, coprécipité dans différentes phases minérales...).

- Les concentrations des différentes especes (dissoutes et Cd adsorbé) sont calculées à partir de CdT 
Tableau II - Valeurs moyennes et écart-type (en italique) des paramètres de sorption utilisés pour les simulations.

Table II - Mean values and standard deviation (in italic) of the sorption parameters used for simulations.

\begin{tabular}{|c|c|c|c|c|c|}
\hline$\ldots$ & [Sites] mol.g ${ }^{-1}$ & $\mathrm{pK}_{\mathrm{a1}}$ & & $\mathrm{pK}_{\mathrm{a} 2}$ & $\log K_{m}$ \\
\hline SEINE & $\begin{array}{l}1.57 E-4 \\
8.67 E-5\end{array}$ & & & $\begin{array}{l}5.59 \\
0.69\end{array}$ & $\begin{array}{l}0.48 \\
0.25\end{array}$ \\
\hline LOIRE & $\begin{array}{l}1.22 E-4 \\
9.97 E-6\end{array}$ & $\begin{array}{l}5.26 \\
0.49\end{array}$ & & $\begin{array}{l}6.59 \\
0.47\end{array}$ & $\begin{array}{c}-1.51 \\
0.66\end{array}$ \\
\hline GIRONDE & $\begin{array}{l}8.78 E-5 \\
2.39 E-5\end{array}$ & $\begin{array}{l}4.96 \\
0.45\end{array}$ & & $\begin{array}{l}6.42 \\
0.46\end{array}$ & $\begin{array}{r}-1.21 \\
0.27\end{array}$ \\
\hline
\end{tabular}

(= Cd dissous mesuré $+\mathrm{Cd}$ particulaire mesuré - Cd particulaire non échangeable). La non prise en compte de cette fraction conduirait à une surestimation des concentrations des différentes espèces calculées par le modèle.

L'approche utilisée pour estimer cette fraction (Gonzalez et al., 2001) est basée sur la comparaison des coefficients de distribution du Cd naturel mesurés lors de différentes campagnes in situ avec les coefficients de distribution de ${ }^{109} \mathrm{C} d$ établis lors d'expériences d'adsorption. La fraction particulaire échangeable est en moyenne de l'ordre de $10 \%$ du Cd particulaire mesuré pour tous les estuaires considérés dans cette étude (Gonzalez et al., 2001). Afin de ne pas multiplier les simulations, c'est cette valeur moyenne qui a été utilisée. Le modèle est sensible à ce paramètre, d'autant plus que la concentration en MES est élevée. La variabilite de ce paramètre au sein de l'estuaire peut être une source non négligeable d'erreurs (cf. 3 ) qu'il convient de réduire par une bonne es- timation, notamment à la limite amont des estuaires (stock de $\mathrm{Cd}$ échangeable entrant) et dans les zones où la teneur en MES est importante (bouchon vaseux par exemple).

Du fait de la variabilité naturelle des paramètres utilisés pour caractériser les propriétés de sorption des particules (concentration en sites actifs de surface, $\mathrm{K}_{\mathrm{a} 1}, \mathrm{~K}_{\mathrm{a} 2}$ et $\mathrm{K}_{\mathrm{m}}$ ) au sein d'un même estuaire, toutes les simulations ont été effectuées avec les jeux des valeurs moyennes, minimales et maximales qui ont été évaluées (tableau II).

A partir de ces données, le modèle distribue la quantité totale de $\mathrm{Cd}$ mesurée ( $\mathrm{Cd}$ dissous + $\mathrm{Cd}$ particulaire - Cd particulaire non échangeable) en Cd adsorbé et différentes espèces dissoutes (tableau I).

\subsection{Validation}

Afin de valider l'approche utilisée (fig. 1) et tester le modèle dans le plus grand nombre de situations possibles, la simulation de la distribution 
Tableau III. - Quelques caractéristiques des estuaires étudiés et conditions hydrologiques lors des campagnes [(1) Cossa et al., 1994, (2) Chiffoleau et al., 1997, (3) Chiffoleau et al., 1998, (4) Boutier et al., 1993, (5) Boutier et al., 2000].

Table III. - Some characteristics of studied estuaries and hydrological conditions during the cruises [(1) Cossa et al., 1994, (2) Chiffoleau et al., 1997, (3) Chiffoleau et al., 1998, (4) Boutier et al., 1993, (5) Boutier et al., 2000].

\section{Caractéristiques}

SEINE

Longueur : $163 \mathrm{~km}$
Débit fluvial :
$440 \mathrm{~m}^{3 /} / \mathrm{s}$ (moyenne)
$2500 \mathrm{~m}^{3} / \mathrm{s}$ (max)

LOIRE

Longueur : $90 \mathrm{~km}$

Débit fluvial :

$850 \mathrm{~m}^{3} / \mathrm{s}$ (moyenne) $3000 \mathrm{~m}^{3} / \mathrm{s}(\max )$

GIRONDE Longueur: $170 \mathrm{~km}$ Débit fluvial : $990 \mathrm{~m}^{3} / \mathrm{s}$ (moyenne) $1500 \mathrm{~m}^{3} / \mathrm{s}$ (max)

\section{Campagnes}

(1) Poses (01/90-05/92)

(2) Marina $6(06 / 96)$

(3) Marina 7 (03/97)

(4) Loire (03/90)

(5) Mecagir (04/99)
Débit $\mathrm{m}^{3} / \mathrm{s}$

[MES] mg/l

$\begin{array}{cc}40-1931 & 2.5-204 \\ 130 & 0-350 \\ 520 & 0.37-874 \\ & \\ 1000 & 12-432\end{array}$

Débit moyen .

$0-667$ de Cd dissous \& particulaire, dans trois estuaires français (la Seine, la Loire et la Gironde), a été comparée aux mesures réalisées lors de différentes saisons et différents régimes hydrologiques (crues, étiages) (Boutier et al., 1993 ; Chiffoleau et al., 1994 ; Cossa et al., 1994 ; Boutier et al., 2000 ; Chiffoleau et al., 2001). Les principales caractéristiques des campagnes sont rassemblées dans le tableau III. II est à noter qu'aucun des paramètres nécessaires n'a fait l'objet d'une calibration à partir des données de terrain, ils sont tous évalués de façon indépendante (cf. 2.2).

De même, la validité de certaines hypothèses du modèle (cf. 2.1.) a été vérifiée expérimentalement, grâce à l'utilisation de $\mathrm{Cd}$ en laboratoire ou a bord des navires lors des campagnes. Le principe de ces expériences repose sur l'ajout de ${ }^{` \mathrm{C}} \mathrm{Cd}$, soit dans les échantillons d'eau brute (eau + particules) prélevée lors des campagnes et marquée à bord, soit dans un “milieu expérimental " constitué de particules prélevées dans les différents estuaires et mises en suspension dans des milieux reconstitués (par exemple : gradient de solutions de $\mathrm{NaCl}$ si l'on veut vérifier l'effet des chlorures).

Après un temps d'incubation fixé, l'échantillon marqué est filtré et l'activité de ${ }^{159} \mathrm{Cd}$ est mesurée dans la phase dissoute et dans les particules recueillies sur le filtre. Grâce à la sensibilité et à la spécificité des méthodes d'analyse radiochimiques (environ 1000 fois plus sensibles que la spectrométrie atomique par exemple), cet outil permet de n'utiliser que de très faibles concentrations (moins de quelques $\mathrm{ng} / \mathrm{l}$ ) de traceurs radioactifs, inférieures aux concen- 
trations naturelles de l'élément stable étudié (comprises, pour $\mathrm{Cd}$ dissous, entre 20 et $100 \mathrm{ng} / \mathrm{l}$ dans les estuaires étudiés).

Les principaux résultats de ces expériences montrent que :

- La cinétique d'adsorption de Cd est relativement rapide (un pseudoéquilibre est atteint en $1 \mathrm{~h}$ environ).

- La réversibilité de l'adsorption est partielle. La quantité de $\mathrm{Cd}$ non-désorbable (ou "lentement" désorbable) devient importante quand le temps d'adsorption augmente. Ce fait a été vérifié en réalisant des expériences de désorption (particules "marquées" avec ${ }^{109} \mathrm{Cd}$ et remises en suspension dans le même milieu sans ${ }^{109} \mathrm{Cd}$ ). Différents temps d'adsorption ont été testés $(1 \mathrm{~h}, 4 \mathrm{~h}, 8 \mathrm{~h}$, $24 \mathrm{~h}, 48 \mathrm{~h}$ ). Après un temps d'adsorption de $24 \mathrm{~h}$ (sans aucun changement des variables physicochimiques au cours du temps de contact), jusqu'à $50 \%$ du ${ }^{103} \mathrm{Cd}$ adsorbé peut devenir non-désorbable "rapidement " (en quelques heures).

Dans la plupart des situations, la non prise en compte de cette réversibilité partielle n'est pas une source d'incertitude majeure pour le modèle du fait que les temps de contact eauparticules (sans modification des conditions physico-chimiques) sont très courts en estuaire (inférieurs à l'heure). De plus, la fraction devenant non-désorbable (à l'échelle de l'heure) ne va représenter qu'une faible partie de la fraction échan- geable (qui ne représente que de l'ordre de $10 \%$ du Cd particulaire total mesuré).

Par contre, dans le cas des simulations à long terme, ce phénomène pourrait être non négligeable et doit être pris en compte, notamment en situation d'étiage dans les zones d'accumulation importante de particules (système crème de vase/bouchon vaseux). Ce fait sera illustré lors d'un exemple du couplage MOCO-SAM3D (cf. 3 ci-après).

- La quantité de Cd associée à la fraction colloïdale est négligeable. Cette hypothèse a pu être vérifiée lors de la campagne Marina 7 , grâce à la mesure des concentrations de Cd dans la fraction colloïdale séparée par ultrafiltration à partir de différents échantillons prélevés le long du gradient de salinité (Chiffoleau et al., 2001). Ces résultats sont confirmés expérimentalement par l'ajout de ${ }^{10^{10}} \mathrm{Cd}$ et de particules identiques dans des échantillons d'eau filtrée $(0,45 \mu \mathrm{m})$ et ultra-filtrée prélevés au cours de la même campagne. Les données obtenues indiquent qu'il n'y a pas de différence significative entre la quantité de ${ }^{153} \mathrm{Cd}$ adsorbée sur les particules, qu'elles soient en suspension dans de l'eau filtrée ou ultra-filtrée.

\section{EXEMPLES DE RÉSULTATS OBTENUS}

Les nombreuses campagnes réalisées par IFREMER ont permis d'ob- 

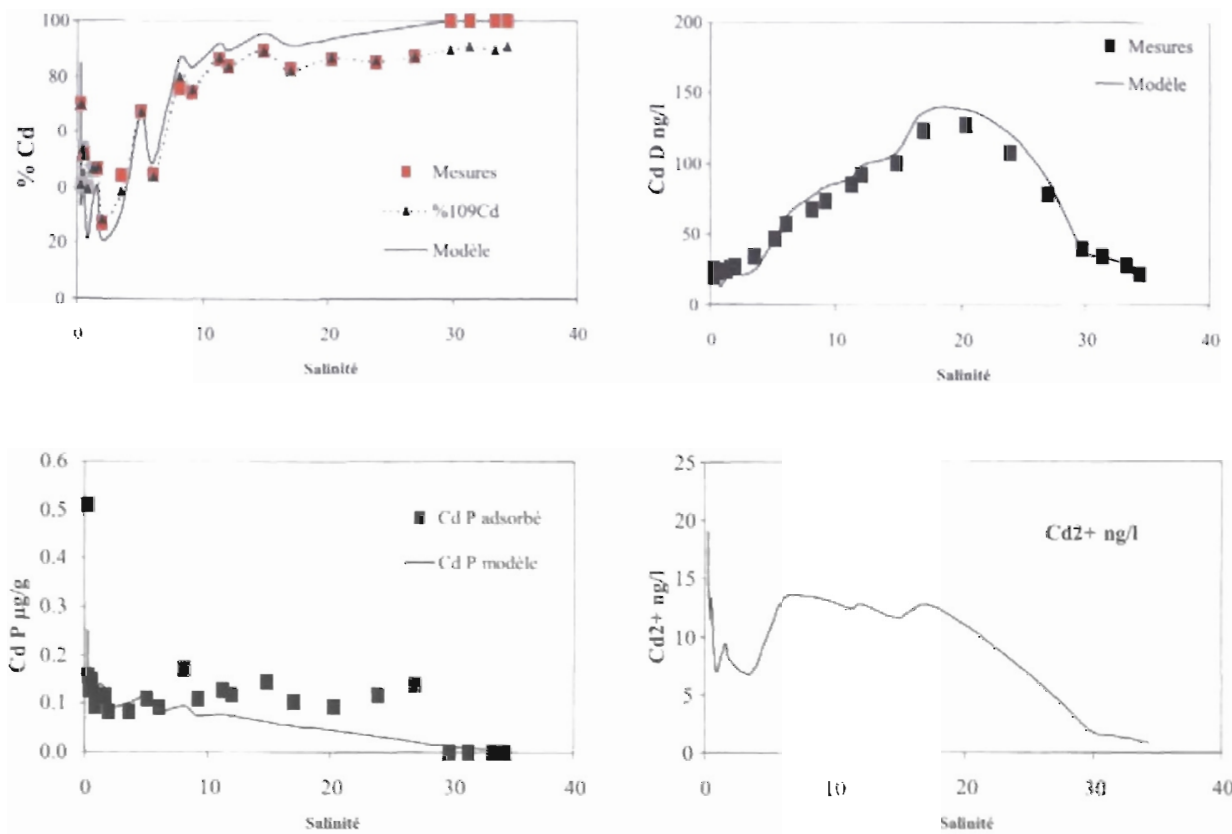

Fig. 4. - Estuaire de la Seine: Comparaison des mesures et des résultats du modèle.

Fig. 4. - Seine estuary : Comparison of measurements and model results.

(a) Simulation d'une campagne longitudinale (MARINA 7 : Chiffoleau el al., 1998) : résultats obtenus avec les paramètres de sorption moyens.

(a) Simulation of a longitudinal cruise (MARINA 7 : Chiffoleau et al., 1998) : results obtained using mean adsorption parameters.

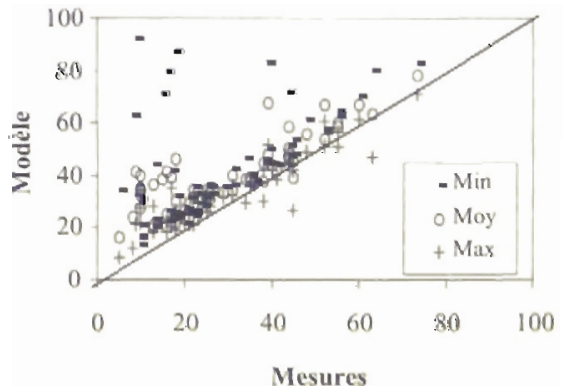

Cd dissous (ng/l)

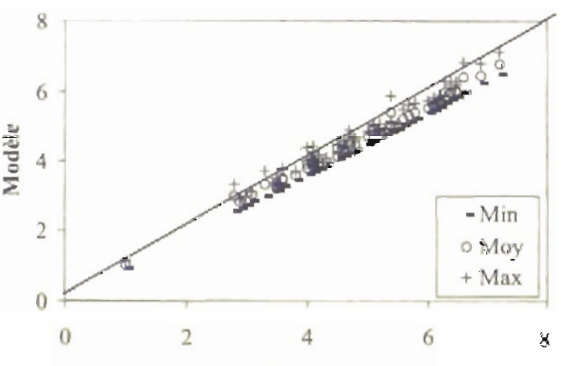

Mesures

Cd particulaire $(\mu \mathrm{g} / \mathrm{g})$

Fig. 4. - Estuaire de la Seine : Comparaison des mesures et des résultats du modèle.

Fig. 4. - Seine estuary : Comparison of measurements and model results.

(b) Station fixe à Poses. Mesures réalisées de 1990 à 1992 (Cossa et al., 1994). Les résultats ont été obtenus avec les paramètres d'adsorption moyens (Moy), minimum (Min) et maximum (Max).

(b) Stationary point of Poses. Measurements from 1990-1992 (Cossa et al., 1994). Results obtained using mean (Moy), minimum (Min) and maximum (Max) adsorption parameters. 

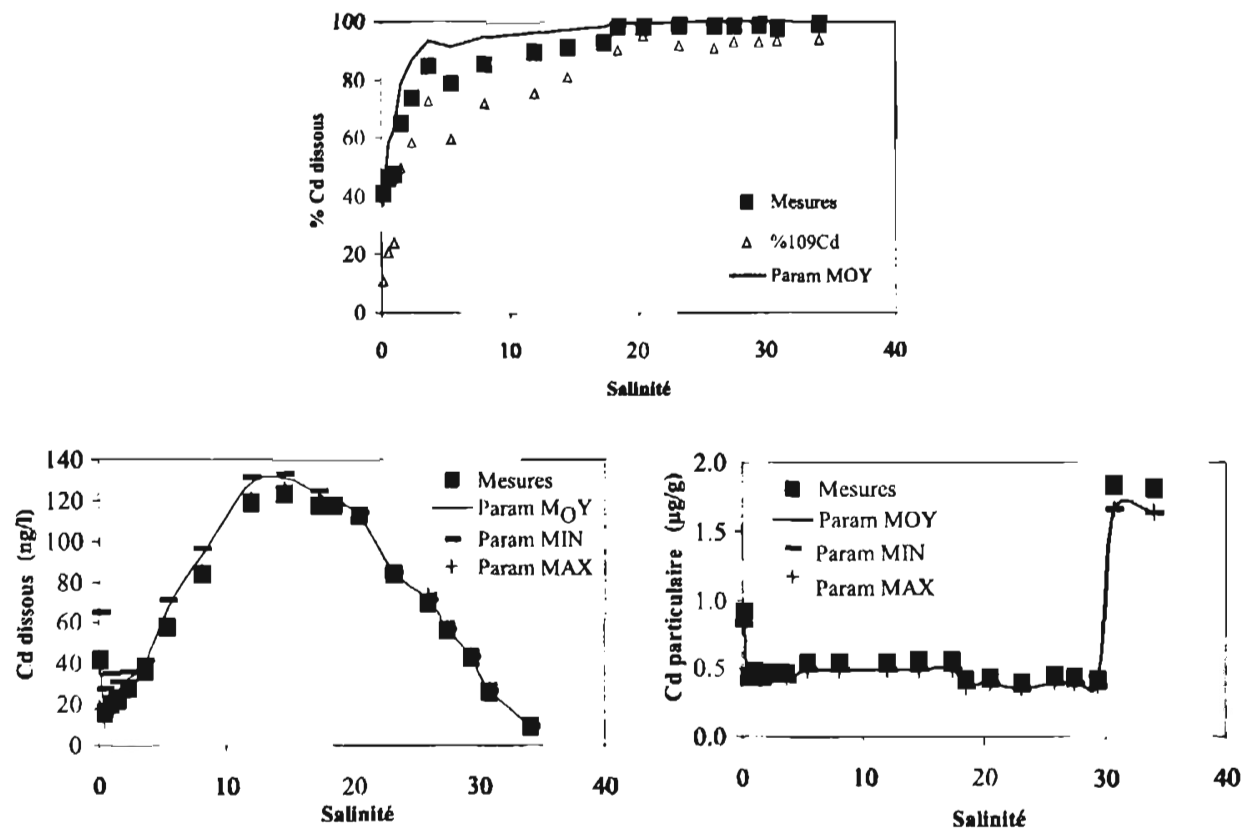

Fig. 5. - Estuaire de la Gironde. Simulation d'une campagne longitudinale (MECAGIR : Boutier et al. 2000). Les résultats ont été obtenus avec les paramètres d'adsorption moyens (Moy), minimum (Min) et maximum (Max).

Fig. 5. - Gironde estuary. Simulation of a longitudinal cruise (MECAGIR : Boutier et al., 2000). Results obtained using mean (Moy), minimum (Min) and maximum (Max) adsorption parameters.

tenir les données nécessaires pour la validation du modèle. Les figures 4 à 6 ne représentent que quelques exemples des simulations réalisées. Pour plus de clarté, les différentes espèces de $\mathrm{Cd}$ dissous (Cd libre, $\mathrm{Cd}$ complexé avec les chlorures, les sulfates ou les hydroxydes) ne sont pas présentées. Les résultats sont exprimés sous forme de $\mathrm{Cd}$ "dissous total " (somme de toutes les espèces dissoutes présentées au tableau I) et de $\mathrm{Cd}$ "particulaire" (qui correspond à la fraction adsorbée calculée par le modèle).
Les résultats montrent que la distribution de Cd mesurée dans les différents estuaires est comparable à celle qui est calculée par le modèle. Malgré ses simplifications, MOCO reproduit de façon satisfaisante le comportement de Cd caractérisé, pour les 3 campagnes simulées, par une solubilisation importante entre 0 et 20 de salinité environ. Ce comportement est en grande partie expliqué par la désorption de Cd liée à la formation de chlorocomplexes du fait de l'augmentation de la salinité. Entre 20 et 35 de salinité, la diminution quasi li- 

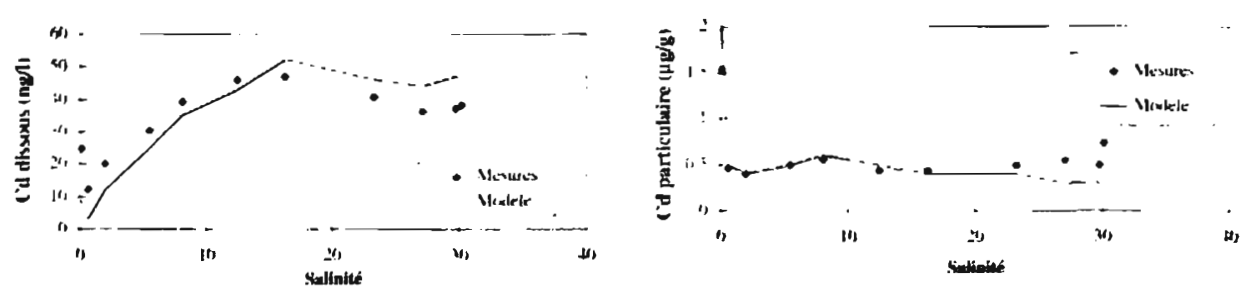

Fig. 6. - Esluaire de la Loire. Simulation d'une campagne longitudinale (LOIRE : Boutier et al., 1993). Les résultats ont été obtenus avec les paramères d'adsorption moyens.

Fig. 6. - Loire estuary. Simulation of a longitudinal cruise (LOIRE : Boutier et al., 1993). Results obtained using mean adsorption parameters.

néaire des concentrations en $\mathrm{Cd}$ dissous est le résultat de la dilution par les eaux marines plus faiblement chargées en Cd (de l'ordre de $10 \mathrm{ng} / \mathrm{l})$. Ce comportement est en accord avec d'autres travaux sur le devenir de Cd en milieu estuarien (Van der Weidjen et al., 1977 ; Elbaz-Poulichet et al., 1987 ; Comans et Van Dijk, 1988 ; Turner, 1996 ; Krapiel et al., 1997). Une interprétation beaucoup plus détaillée de l'évolution des concentrations en Cd (dissous et particulaire) dans les trois estuaires est donnée dans les articles portant sur les campagnes qui ont servi à la validation du modèle (Boutier et al., 1993 ; Chiffoleau et al., 1994 ; Cossa et al., 1994 ; Boutier et al., 2000 ; Chiffoleau et al., 2001).

Le modèle évalue les différentes espèces dissoutes présentes dans le milieu et peut ainsi permettre d'avoir une meilleure vision de la biodisponibilité (espèce $\mathrm{Cd}^{2 \cdot}$ par exemple) du contaminant qui n'est pas liée directement à la concentration totale. A titre d'exemple, nous avons représenté la concentration en $\mathrm{Cd}^{z \cdot} \mathrm{Cal}-$ culée par MOCO pour la campagne réalisée dans l'estuaire de la Seine (fig. 4 a). La comparaison indique que les concentrations maximales en $\mathrm{Cd}$ dissous (vers 20 de salinité) ne correspondent pas aux concentrations maximales en $\mathrm{Cd}^{2}$. (vers 0 et entre 5 et 20 de salinité). Malheureusement, aucune mesure de la concentration en $\mathrm{Cd}^{2-}$ n'a été réalisée. II faut noter que ce type de mesure est difficile et n'est pas effectué en routine.

Dans les figures 4 a et 5 sont comparés, exprimés, sous forme de pourcentage dissous, les mesures de $\mathrm{Cd}$ naturel, le pourcentage de ${ }^{109} \mathrm{Cd}$ resté sous forme dissoute (après ajout d'une quantité connue de ${ }^{150} \mathrm{Cd}$ à des échantillons d'eau brute prélevés le long de l'estuaire) et les résultats du modèle. II est à noter un assez bon accord entre ces trois jeux de données obtenus de façon indépendante.

II peut exister des écarts importants entre les mesures et le modèle, en particulier pour les faibles salinités (où l'importance de la complexation 
par les chlorures est moindre) et les fortes concentrations en matières en suspension. Dans ces parties de l'estuaire, le modèle est très sensible aux paramètres de sorption et à la quantité de Cd particulaire échangeable. Une surestimation (sous estimation) des concentrations en $\mathrm{Cd}$ dissous peut être liée à une fraction de $C d$ particulaire échangeable trop importante (faible) et/ou à une valeur des paramètres de sorption trop faible (forte). Cette sensibilité est illustrée par les simulations réalisées à Poses (fig. 4 b) qui montrent une surestimation de Cd d'autant plus forte que les paramètres de sorption utilisés sont faibles (= capacités d'adsorption réduites). Par rapport au reste de l'estuaire de la Seine, cette station correspondant à la limite de la marée dynamique, se caractérise par une salinité quasi nulle et des teneurs en MES pouvant être importantes par fort débit. Les plus fortes surestimations de $\mathrm{Cd}$ dissous correspondent aux prélèvements pour lesquels les concentrations en MES mesurées sont élevées (comprises entre 0,1 et $0,2 \mathrm{~g} / \mathrm{l})$. Lors de la campagne en Gironde, c'est uniquement dans la zone de salinité très faible (inférieure à $0,5)$, où les teneurs en MES sont les plus élevées (de 0,4 à $0,6 \mathrm{~g} / \mathrm{l}$ ), que $\mathrm{Cd}$ dissous est surestimé par le modèle (fig. 5).

Avec des paramètres de sorption moyens, le modèle reproduit moins bien les concentrations en Cd particulaire pour les salinités comprises entre 10 et 28 en Seine (fig. 4 a) et supérieures à 30 en Loire (fig. 6). Le modèle ayant été utilisé avec des paramètres de sorption constants tout le long de l'estuaire, une explication possible serait la présence locale de particules dont les capacités de sorption vis-à-vis de $\mathrm{Cd}$ seraient plus élevées (matériel phytoplanctonique par exemple).

Afin de mieux comprendre les processus qui affectent le transfert et la spéciation de Cd, MOCO a été couplé au modèle de transport multivariables (SAM 3D). Cet outil qui est décrit de façon plus précise par ailleurs (Thouvenin et al., 1999) simule le mouvement des masses d'eau, le transport et le mélange de toute variable dissoute ou particulaire conservative. Les résultats du couplage réalisé pour l'estuaire de la Seine sont présentés dans Gonzalez et al. (1999; 2001). Les applications de ce type d'outil, après validation, sont nombreuses, par exemple: hiérarchiser les différents processus contrôlant la dynamique estuarienne d'un contaminant comme $\mathrm{Cd}$; estimer des flux à l'océan ; prévoir les effets d'une modification du systeme estuarien sur le cycle de Cd.

Mais le couplage nécessite souvent, en plus d'un modèle de transport et d'un modèle géochimique validés, la prise en compte d'autres processus. A titre d'illustration, la figure 7 présente les résultats du couplage comparés aux mesures effectuées en Seine lors de la Campagne Marina 6 (Chiffoleau et al., 1997). Après 6 mois de simulation (débutée en janvier 96), on obtient (en prenant uniquement en compte le 
A

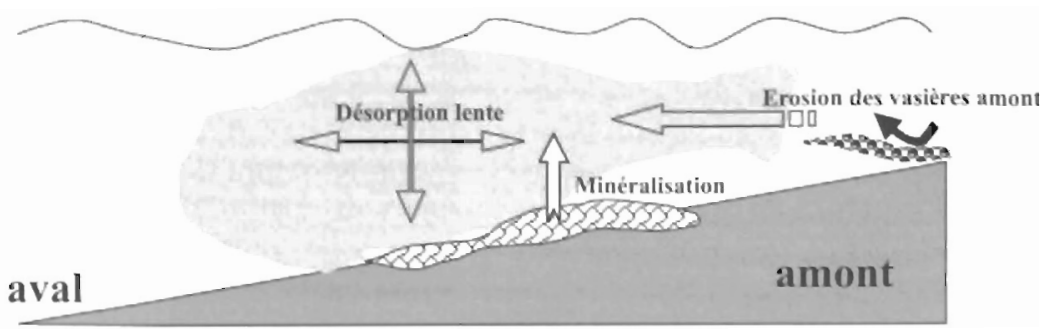

B

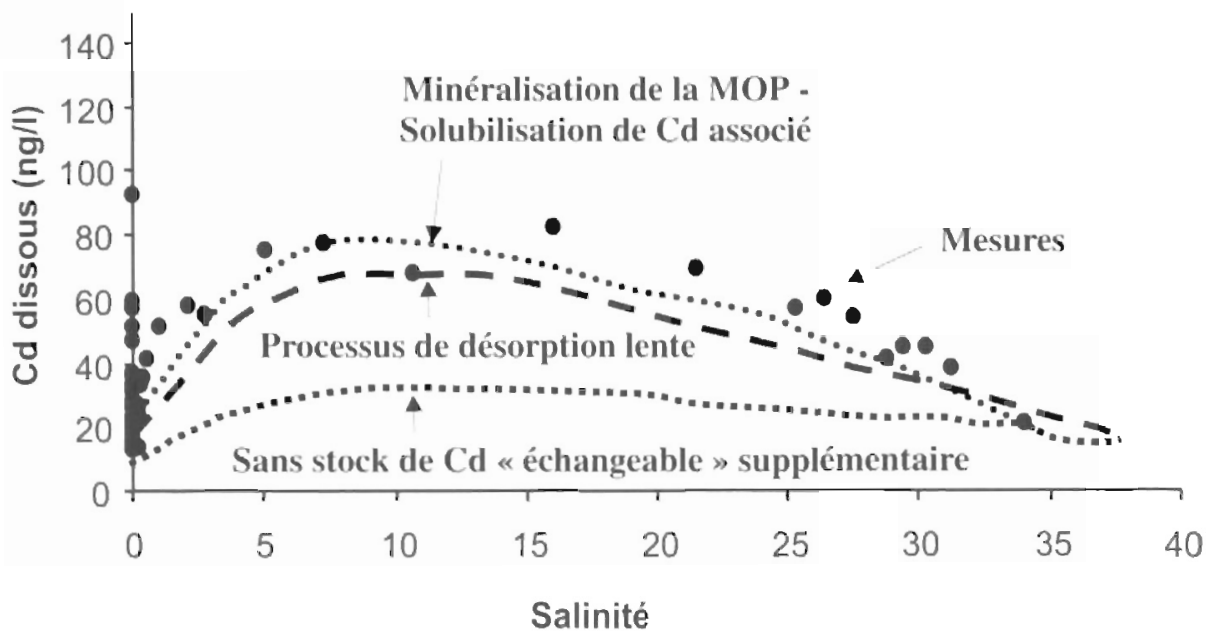

Fig. 7. - Couplage SAM3D-MOCO : Prise en compte de processus supplémentaires (A). Exemple (B) de résultats obtenus pour la simulation de la campagne MARINA 6 (Juin 1996-étiage).

Fig. 7. - SAMBD-MOCO coupling : Taking into account of additional processes (A). Example (B) of results obtained for the simulation of the MARINA 6 cruise (June 1996-low river flow).

Cd échangeable introduit à la limite amont) de très faibles concentrations en Cd dissous par rapport aux mesures. La majeure partie du Cd particulaire échangeable est introduit dans l'estuaire lors des crues et est "rapidement " désorbé par le modèle au cours de l'hiver et du printemps. Il doit donc exister un stock de Cd échangeable supplémentaire qui ne peut être issu de ce stock.

Nous avons testé différents processus pouvant compenser ce déficit en $\mathrm{Cd}$ dissous (fig. 7) : la désorption 
lente de $\mathrm{Cd}$ à partir du stock particulaire introduit dans l'estuaire et dépourvu de la fraction rapidement échangeable; le retour dans la colonne d'eau, lors des périodes d'érosion, du cadmium solubilisé lors de la minéralisation de la matière organique particulaire dans la colonne sédimentaire (diagénése précoce). La simulation de ces processus a été réalisée en prenant des paramètres "réalistes" (cinétique de désorption lente, concentration de $\mathrm{Cd}$ dans les eaux interstitielles...) issus de la bibliographie. Les détails de ces simulations sont donnés dans Gonzalez et al., (2001). Avec ces différents processus, les résultats obtenus deviennent tout à fait comparables aux mesures.

D'autres processus pourraient aussi combler le stock de Cd échangeable manquant : la minéralisation du $\mathrm{Cd}$ dans le système bouchon vaseux/crème de vase; les apports par les vasières latérales amont lors de leur érosion. En Loire et en Gironde, du fait de la présence d'un bouchon vaseux et de zones d'accumulation de vase importantes, ces types de processus doivent probablement aussi être pris en compte.

\section{CONCLUSION ET PERSPECTIVES}

L'application d'un modèle de complexation de surface pour simuler le comportement et la spéciation de $\mathrm{Cd}$ en milieu estuarien montre l'utilité et les limites de cette approche.
Les résultats montrent que les processus pris en compte expliquent assez bien la distribution dissous/ particulaire de cet élément. Cependant, une mauvaise évaluation des paramètres de sorption utilisés par le modèle peut être la cause d'écarts importants entre le modèle et les mesures de terrain, notamment aux faibles salinités quand les concentrations en MES sont importantes. De plus, dans certaines parties des estuaires, des processus non pris en compte pourraient avoir de l'importance sur le devenir de $\mathrm{Cd}$, par exemple: l'activité phytoplanctonique à certaines périodes de l'année, des processus de dissolution et de précipitation dans les zones de turbidité maximale où les gradients redox peuvent être très importants.

L'utilisation de ce type de modèle dans un but "prédictif " implique le couplage à un modèle de transport multivariables qui reproduit avec une bonne précision l'évolution spatiotemporelle des variables nécessaires aux calculs de spéciation (salinité, MES...). Les premiers résultats ont montré (Gonzalez et al., 2001) que des simulations à long terme nécessitent une bonne évaluation des flux de contaminant qui entrent dans l'estuaire (évolution temporelle en fonction du débit fluvial, flux particulaire échangeable...) et l'intégration d'autres processus (désorption lente, flux à l'interface eau-sédiment, apports des vasières latérales...). 


\section{REMERCIEMENTS}

Ce travail a été mené dans le cadre du projet IFREMER " Modélisation du cycle des contaminants" et du programme “Seine-Aval" (financé par le Conseil Régional de Haute-Normandie, l'Agence de l'eau Seine-Normandie et les industriels de Haute-Normandie). Nous remercions: B. Boutier, J.F. Chiffoleau et D. Cossa qui grâce à leurs données ont permis la validation du modèle; E. Chartier et D. Auger pour leur aide précieuse lors des campagnes.

\section{RÉFÉRENCES BIBLIOGRAPHIQUES}

Boutier B., Chiffoleau J.F., Auger D. \& Truquet I., 1993. Influence of the Loire river on dissolved lead and cadmium concentrations in coastal waters of Brittany. Estuarine Coastal Shelf Science, $36: 133-145$.

Boutier B., Chiffoleau J.F., Gonzalez J.L., Lazure P., Auger D. \& Truquet I., 2000. Influence of the Gironde Estuary outputs on cadmium concentrations in the coastal waters. Consequences on the Marennes Oleron Bay (France). Oceanologica Acta, 23 (7) : 745-757.

Chiffoleau J.F., Cossa D., Auger D. \& Truquet I., 1994. Trace metal distribution, partition and fluxes in the Seine estuary (France) in low discharge regime. Marine Chemistry, 47 : 145-158.

Chiffoleau J.F., Auger D., Chartier E. \& Truquet I.. 1997. Identification et devenir des apports intra-estuariens de métaux traces dans l'estuaire de la Seine. Programme Scientifique "Seine Aval". Rapport final 1996. Theme "Dynamique des contaminants": 5-23.
Chiffoleau J.F., Auger D. \& Chartier E., 1998. Contribution à la modélisation du comportement du cadmium dans l'estuaire de la Seine. Étude des phénomènes de sorption et du rôle des colloïdes. Programme Scientifique "Seine Aval". Rapport final 1997. Thème "Dynamique des contaminants ": 12-27.

Chiffoleau J.F., Claisse D., Cossa D., Ficht A., Gonzalez J.L., Guyot T., Michel P., Miramand P., Oger C. \& Petit $F$., 2001. La contamination métallique. IFREMER ed. Programme Scientifique "Seine Aval ", fascicule n" $8: 39$ pp.

Chiffoleau J.F., Auger D., Chartier E., Michel P., Truquet I., Ficht A., Gonzalez J.L. \& Romana L.A., 2001. Spatiotemporal changes in cadmium contamination in the Seine Estuary (France). Estuaries, 24, 6B : 1029-1040.

Comans R.N.J. \& Van Dijk C.P.J.. 1988. Role of complexation processes in cadmium mobilization during estuarine mixing. Nature, $336:$ 151-154.

Cossa D., Meybeck M., Idlafkin Z. \& Bombled B., 1994. Étude pilote des apports en contaminants par la Seine. Rapport IFREMER, DEL 94-13:151 pp.

Elbaz-Poulichet F., Huang W.W., Martin J.M. \& Zhu J.X., 1987. Dissolved cadmium behaviour in some selected french and chinese estuaries. Consequences on cadmium supply to the ocean. Marine. Chemistry, 22 : 125-136.

Gonzalez J.L., Thouvenin B., Chiffoleau J.F. \& Miramand P., 1999. Le cadmium: comportement d'un contaminant métallique en estuaire. IFREMER ed. Programme Scientifique "Seine Aval ", fascicule n" $10: 31 \mathrm{pp}$.

Gonzalez J.L., Thouvenin B., Dange C.. Fiandrino A. \& Chiffoleau J.F., 2001. Modeling of $\mathrm{Cd}$ speciation and dynamics in the Seine estuary (France). Estuaries, 24, 6B : 1041-1055.

Gulmini M., Zelano V., Daniele P.G., Prenesti E. \& Ostacoli G., 1996. Acid-Base 
properties of a river sediment : potentiometric titrations. Analytical Chimica Acta, 329: 33-39.

Krapile A.M.L., Chiffoleau J.F., Matin J.M. \& Morel F.M.M., 1997. Geochemistry of trace metals in the Gironde estuary. Geochimica Cosmochimica Acta, 61 : 1421-1436.

Stumm W., 1992. Chemistry of the Solid-Water interface. Wiley-Interscience ed. : 428 pp.

Thouvenin B., Billen G., Even S., Fischer J.C., Gonzalez J.L., Le Hir P., Loizeau V., Mouchel J.M., Olivier C. \& Silva Jacinto R., 1999. Les modèles : outils de connaissance et de gestion. IFREMER ed. Programme Scientifique "Seine Aval ", fascicule n"16:32 pp.
Turner A., 1996. Trace metal partitioning in estuaries: importance of salinity and particle concentration. Marine Chemistry, 54 : 27-39.

Van der Weidjen C.H., Arnoldus M.J.H.L. \& Meurs C.J., 1977. Desorption of metals from suspended material in the Rhine estuary. Netherland. Journal of Sea Research, 11 : 130-145.

Wang F., Chen J. \& Forsling W., 1997. Surface properties of natural aquatic sediments. Water research, $31, n^{\circ} 7$ : 796-1800.

Westall J.C., 1982. FITEQL - A computer program for determination of chemical equilibrium constants from experimental data. Dep. of Chemistry Rep. 82-01. Oregon State University, Corvallis. 